\title{
Recommendation for Key-Derivation Methods in Key-Establishment Schemes
}

Elaine Barker

Lily Chen

Richard Davis

This publication is available free of charge from: https://doi.org/10.6028/NIST.SP.800-56Cr2

\section{O M P U TER SE C UR I T Y}




\title{
NIST Special Publication 800-56C \\ Revision 2
}

\section{Recommendation for Key-Derivation Methods in Key-Establishment Schemes}

\author{
Elaine Barker \\ Lily Chen \\ Computer Security Division \\ Information Technology Laboratory
}

Richard Davis

National Security Agency

This publication is available free of charge from: https://doi.org/10.6028/NIST.SP.800-56Cr2

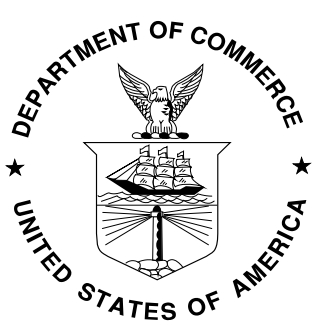

U.S. Department of Commerce Wilbur L. Ross, Jr., Secretary

National Institute of Standards and Technology Walter Copan, NIST Director and Under Secretary of Commerce for Standards and Technology 


\title{
Authority
}

This publication has been developed by NIST in accordance with its statutory responsibilities under the Federal Information Security Modernization Act (FISMA) of 2014, 44 U.S.C. § 3551 et seq., Public Law (P.L.) 113-283. NIST is responsible for developing information security standards and guidelines, including minimum requirements for federal information systems, but such standards and guidelines shall not apply to national security systems without the express approval of appropriate federal officials exercising policy authority over such systems. This guideline is consistent with the requirements of the Office of Management and Budget (OMB) Circular A-130.

Nothing in this publication should be taken to contradict the standards and guidelines made mandatory and binding on federal agencies by the Secretary of Commerce under statutory authority. Nor should these guidelines be interpreted as altering or superseding the existing authorities of the Secretary of Commerce, Director of the OMB, or any other federal official. This publication may be used by nongovernmental organizations on a voluntary basis and is not subject to copyright in the United States. Attribution would, however, be appreciated by NIST.

\section{National Institute of Standards and Technology Special Publication 800-56C Revision 2 Natl. Inst. Stand. Technol. Spec. Publ. 800-56C Rev. 2, 41 pages (August 2020)} CODEN: NSPUE2

This publication is available free of charge from: https://doi.org/10.6028/NIST.SP.800-56Cr2

Certain commercial entities, equipment, or materials may be identified in this document in order to describe an experimental procedure or concept adequately. Such identification is not intended to imply recommendation or endorsement by NIST, nor is it intended to imply that the entities, materials, or equipment are necessarily the best available for the purpose.

There may be references in this publication to other publications currently under development by NIST in accordance with its assigned statutory responsibilities. The information in this publication, including concepts and methodologies, may be used by federal agencies even before the completion of such companion publications. Thus, until each publication is completed, current requirements, guidelines, and procedures, where they exist, remain operative. For planning and transition purposes, federal agencies may wish to closely follow the development of these new publications by NIST.

Organizations are encouraged to review all draft publications during public comment periods and provide feedback to NIST. Many NIST cybersecurity publications, other than the ones noted above, are available at https://csrc.nist.gov/publications.

\section{Comments on this publication may be submitted to:}

\author{
National Institute of Standards and Technology \\ Attn: Computer Security Division, Information Technology Laboratory \\ 100 Bureau Drive (Mail Stop 8930) Gaithersburg, MD 20899-8930 \\ Email: 800-56C Comments@nist.gov
}

All comments are subject to release under the Freedom of Information Act (FOIA). 


\title{
Reports on Computer Systems Technology
}

The Information Technology Laboratory (ITL) at the National Institute of Standards and Technology (NIST) promotes the U.S. economy and public welfare by providing technical leadership for the Nation's measurement and standards infrastructure. ITL develops tests, test methods, reference data, proof of concept implementations, and technical analyses to advance the development and productive use of information technology. ITL's responsibilities include the development of management, administrative, technical, and physical standards and guidelines for the cost-effective security and privacy of other than national security-related information in federal information systems. The Special Publication 800-series reports on ITL's research, guidelines, and outreach efforts in information system security, and its collaborative activities with industry, government, and academic organizations.

\begin{abstract}
This Recommendation specifies techniques for the derivation of keying material from a shared secret established during a key-establishment scheme defined in NIST Special Publications 800$56 \mathrm{~A}$ or $800-56 \mathrm{~B}$.
\end{abstract}

\section{Keywords}

Expansion; extraction; extraction-then-expansion; hash function; key derivation; key establishment; message authentication code. 


\section{Acknowledgements}

The authors gratefully acknowledge the contributions on this and previous versions of this document by their NIST colleagues (Quynh Dang, Sharon Keller, John Kelsey, Allen Roginsky, Meltem Sonmez Turan, Apostol Vassilev, and Tim Polk) and by Miles Smid, formerly of Orion Security Solutions.

The authors also gratefully appreciate the thoughtful and instructive comments received during the public comment periods, which helped to improve the quality of this publication.

\section{Conformance Testing}

Conformance testing for implementations of the functions that are specified in this publication will be conducted within the framework of the Cryptographic Algorithm Validation Program (CAVP) and the Cryptographic Module Validation Program (CMVP). The requirements on these implementations are indicated by the word "shall." Some of these requirements may be out of scope for CAVP or CMVP validation testing and are therefore the responsibility of entities using, implementing, installing, or configuring applications that incorporate this Recommendation. 


\section{Patent Disclosure Notice}

NOTICE: The Information Technology Laboratory (ITL) has requested that holders of patent claims whose use may be required for compliance with the guidance or requirements of this publication disclose such patent claims to ITL. However, holders of patents are not obligated to respond to ITL calls for patents and ITL has not undertaken a patent search in order to identify which, if any, patents may apply to this publication.

As of the date of publication and following call(s) for the identification of patent claims whose use may be required for compliance with the guidance or requirements of this publication, no such patent claims have been identified to ITL.

No representation is made or implied by ITL that licenses are not required to avoid patent infringement in the use of this publication. 


\section{Table of Contents}

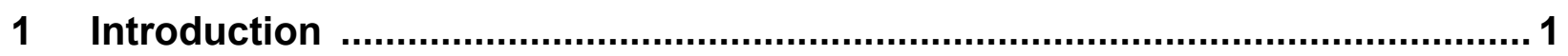

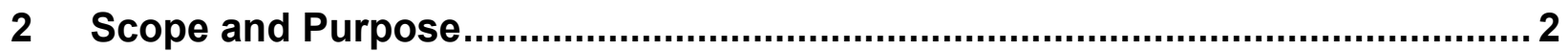

3 Definitions, Symbols and Abbreviations …................................................. 3

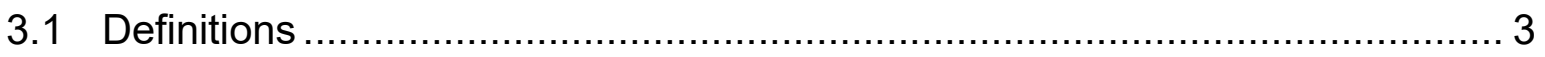

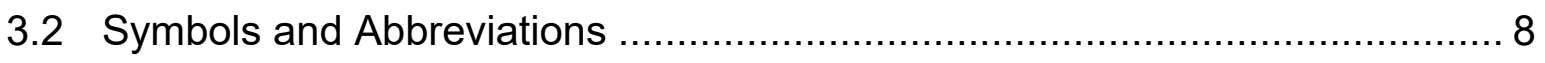

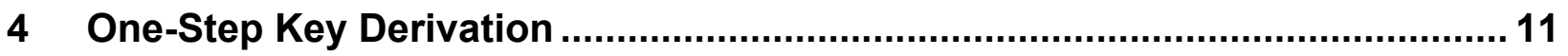

4.1 Specification of Key-Derivation Functions.............................................. 11

4.2 The Auxiliary Function $\mathrm{H}(x)$ and Related Parameters .............................. 15

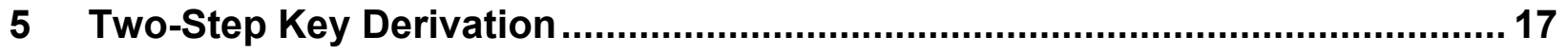

5.1 Specification of Key-Derivation Procedure ............................................. 17

5.2 The Auxiliary MAC Algorithm and Related Parameters ............................ 20

5.3 Randomness Extraction followed by Multiple Key Expansions ..................... 21

6 Application-Specific Key-Derivation Methods................................................. 25

7 Selecting Hash Functions and MAC Algorithms .............................................. 26

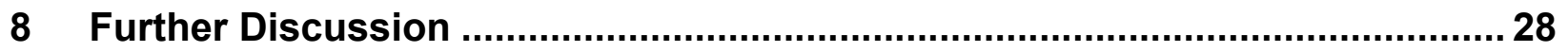

8.1 Using a Truncated Hash Function........................................................ 28

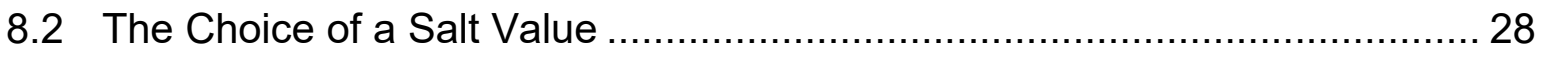

8.3 MAC Algorithms used for Extraction and Expansion.................................. 28

8.4 Destruction of Sensitive Locally Stored Data ............................................ 29

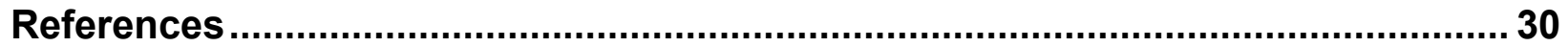

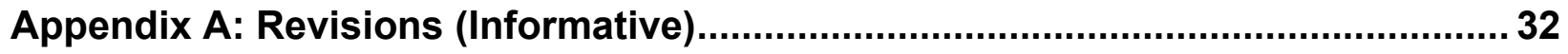

A.1 The Original Version of SP 800-56C ................................................. 32

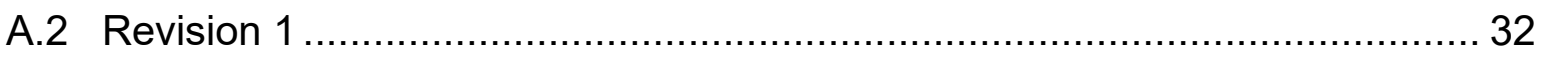

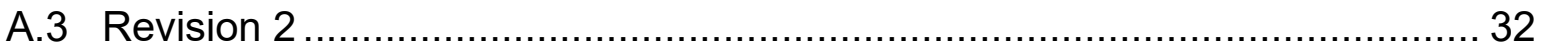




\section{List of Figures}

Figure 1: The Extraction-then-Expansion Key-Derivation Procedure …............. 17

Figure 2: Randomness Extraction followed by Multiple Key Expansions ........... 22

\section{List of Tables}

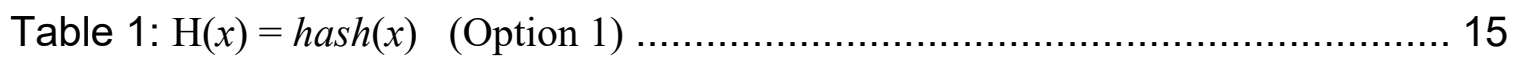

Table 2: H(x) = HMAC-hash(salt, $x) \quad($ Option 2) ................................................... 15

Table 3: H(x) = KMAC\#(salt, $x$, H_outputBits, "KDF”) (Option 3) ........................ 16

Table 4: MAC( salt, $Z, \ldots$ ) = HMAC-hash ( salt, $Z$ ) (For Randomness Extraction) ... 20

Table 5: MAC( salt, $Z, \ldots)=$ AES- $N$-CMAC ( salt,$Z$ ) (For Randomness Extraction). 21 


\section{Introduction}

During the execution of a public key-based key-establishment scheme specified in either of the National Institute of Standards and Technology (NIST) Special Publications [SP 800-56A] ${ }^{1}$ or [SP 800-56B], ${ }^{2}$ a key-derivation method may be required to obtain secret cryptographic keying material. This Recommendation specifies the key-derivation methods that can be used, as needed, in those key-establishment schemes. The keying material derived using these methods shall be computed in its entirety before outputting any portion of it and shall only be used as secret keying material, such as a symmetric key used for data encryption or message integrity, a secret initialization vector, or, perhaps, a key-derivation key that will be used to generate additional keying material (possibly using a different derivation process; see [SP 800-108] ${ }^{3}$ ). The derived keying material shall not be used as a key stream for a stream cipher.

\footnotetext{
${ }^{1}$ SP 800-56A, Recommendation for Pair-Wise Key-Establishment Schemes Using Discrete Logarithm Cryptography

${ }^{2}$ SP 800-56B, Recommendation for Pair-Wise Key-Establishment Schemes Using Integer Factorization Cryptography

${ }^{3}$ SP 800-108, Recommendation for Key Derivation Using Pseudorandom Functions (Revised)
} 


\section{Scope and Purpose}

This Recommendation specifies two categories of key-derivation methods that can be employed, as required, to derive keying material from a shared secret $Z$ generated during the execution of a key-establishment scheme specified in [SP 800-56A] or [SP 800-56B].

In addition to the currently approved techniques for the generation of the shared secret $Z$ as specified in SP 800-56A and SP 800-56B, this Recommendation permits the use of a "hybrid" shared secret of the form $Z^{\prime}=Z \| T$, a concatenation consisting of a "standard" shared secret $Z$ that was generated during the execution of a key-establishment scheme (as currently specified in [SP 800-56A] or [SP 800-56B]) followed by an auxiliary shared secret $T$ that has been generated using some other method. The content, format, length, and method used to generate $T$ must be known and agreed upon by all parties that will rely upon the derived keying material, as well as by any agents trusted to act on their behalf. The key-derivation methods specified in this Recommendation will process a hybrid $Z^{\prime}$ in the same way they process a standard $Z$. Therefore, for simplicity of notation and exposition, any shared secret denoted by the symbol $Z$ in the remainder of this Recommendation can be of either the "standard" or "hybrid" variety.

The first category of specified key-derivation methods consists of a family of one-step keyderivation functions that derive keying material of a desired length from a shared secret that was generated during the execution of a key-establishment scheme (and possibly other information as well).

The second category consists of an extraction-then-expansion key-derivation procedure that involves two steps:

1) Randomness extraction, to obtain a single cryptographic key-derivation key from a shared secret generated during the execution of a key-establishment scheme.

2) Key expansion, to derive keying material of the desired length from that key-derivation key and other information. Since NIST's [SP 800-108] specifies several families of keyderivation functions that are approved for deriving additional keying material from a given cryptographic key-derivation key, those functions are employed in the second (keyexpansion) step of these two-step procedures.

In addition to the key-derivation methods whose specifications are provided in this document, [ $\underline{\mathrm{SP}}$ $\underline{800-135}]^{4}$ describes several variants (of both the one-step and two-step methods) that are approved for specific applications.

${ }^{4}$ SP 800-135 Rev. 1, Recommendation for Existing Application-Specific Key Derivation Functions 


\section{Definitions, Symbols, and Abbreviations}

\subsection{Definitions}

\begin{tabular}{|c|c|}
\hline Algorithm & $\begin{array}{l}\text { A clearly specified mathematical process for computation; a set of } \\
\text { rules that, if followed, will give a prescribed result. }\end{array}$ \\
\hline Approved & $\begin{array}{l}\text { An algorithm or technique that is either 1) specified in a Federal } \\
\text { Information Processing Standard (FIPS) or NIST } \\
\text { Recommendation, 2) adopted in a FIPS or NIST Recommendation, } \\
\text { or 3) specified in a list of NIST-approved security functions. }\end{array}$ \\
\hline Big-endian & $\begin{array}{l}\text { The property of a byte string having its bytes positioned in order of } \\
\text { decreasing significance. In particular, the leftmost (first) byte is the } \\
\text { most significant (containing the most significant eight bits of the } \\
\text { corresponding bit string), and the rightmost (last) byte is the least } \\
\text { significant (containing the least significant eight bits of the } \\
\text { corresponding bit string). } \\
\text { For the purposes of this Recommendation, it is assumed that the } \\
\text { bits within each byte of a big-endian byte string are also positioned } \\
\text { in order of decreasing significance (beginning with the most } \\
\text { significant bit in the leftmost position and ending with the least } \\
\text { significant bit in the rightmost position). }\end{array}$ \\
\hline Bit length & $\begin{array}{l}\text { The number of bits in a bit string (e.g., the bit length of the string } \\
0110010101000011 \text { is sixteen bits). The bit length of the empty } \\
\text { (i.e., null) string is zero. }\end{array}$ \\
\hline Bit string & $\begin{array}{l}\text { An ordered sequence of bits (represented as } 0 \mathrm{~s} \text { and } 1 \mathrm{~s} \text { ). Unless } \\
\text { otherwise stated in this document, bit strings are depicted as } \\
\text { beginning with their most significant bit (shown in the leftmost } \\
\text { position) and ending with their least significant bit (shown in the } \\
\text { rightmost position). For example, the most significant (leftmost) bit } \\
\text { of } 0101 \text { is } 0 \text {, and its least significant (rightmost) bit is } 1 \text {. If } \\
\text { interpreted as the } 4 \text {-bit binary representation of an unsigned integer, } \\
0101 \text { corresponds to the number five. }\end{array}$ \\
\hline Byte & A bit string consisting of eight bits. \\
\hline Byte length & $\begin{array}{l}\text { The number of consecutive (non-overlapping) bytes in a byte } \\
\text { string. For example, } 0110010101000011=01100101 \| 01000011 \text { is } \\
\text { two bytes long. The byte length of the empty string is zero. }\end{array}$ \\
\hline Byte string & $\begin{array}{l}\text { An ordered sequence of bytes, beginning with the most significant } \\
\text { (leftmost) byte and ending with the least significant (rightmost) }\end{array}$ \\
\hline
\end{tabular}




\begin{tabular}{|c|c|}
\hline & $\begin{array}{l}\text { byte. Any bit string whose bit length is a multiple of eight can be } \\
\text { viewed as the concatenation of an ordered sequence of bytes (i.e., a } \\
\text { byte string). For example, the bit string } 0110010101000011 \text { can be } \\
\text { viewed as a byte string since it is the concatenation of two bytes: } \\
01100101 \text { followed by } 01000011 \text {. }\end{array}$ \\
\hline Concatenation & $\begin{array}{l}\text { As used in this Recommendation, the concatenation } X \| Y \text { of bit } \\
\text { string } X \text { followed by bit string } Y \text { is the ordered sequence of bits } \\
\text { formed by appending } Y \text { to } X \text { in such a way that the leftmost (i.e., } \\
\text { initial) bit of } Y \text { follows the rightmost (i.e., final) bit of } X \text {. }\end{array}$ \\
\hline Cryptographic key (Key) & $\begin{array}{l}\text { A parameter used with a cryptographic algorithm that determines } \\
\text { its operation. }\end{array}$ \\
\hline $\begin{array}{l}\text { Estimated maximum } \\
\text { security strength }\end{array}$ & $\begin{array}{l}\text { An estimate of the largest security strength that can be attained by } \\
\text { a cryptographic mechanism given the explicit and implicit } \\
\text { assumptions that are made regarding its implementation and } \\
\text { supporting infrastructure (e.g., the algorithms employed, the } \\
\text { selection of associated primitives and/or auxiliary functions, the } \\
\text { choices for various parameters, the methods of generation and/or } \\
\text { protection for any required keys, etc.). The estimated maximum } \\
\text { security strengths of various approved cryptographic mechanisms } \\
\text { are provided in [SP 800-57]. }\end{array}$ \\
\hline Hash function & $\begin{array}{l}\text { A function that maps a bit string of arbitrary length to a fixed-length } \\
\text { bit string. Depending upon the relying application, the security } \\
\text { strength that can be supported by a hash function is typically } \\
\text { measured by the extent to which it possesses one or more of the } \\
\text { following properties: } \\
\text { 1. (Collision resistance) It is computationally infeasible to find } \\
\text { any two distinct inputs that map to the same output. } \\
\text { 2. (Preimage resistance) Given a randomly chosen target } \\
\text { output, it is computationally infeasible to find any input that } \\
\text { maps to that output. (This property is called the one-way } \\
\text { property.) } \\
\text { 3. (Second preimage resistance) Given one input value, it is } \\
\text { computationally infeasible to find a second (distinct) input } \\
\text { value that maps to the same output as the first value. } \\
\text { This Recommendation uses the strength of the preimage resistance } \\
\text { of a hash function as a contributing factor when determining the } \\
\text { security strength provided by a key-derivation method. }\end{array}$ \\
\hline
\end{tabular}




\begin{tabular}{|c|c|}
\hline & $\begin{array}{l}\text { Approved hash functions are specified in [FIPS 180] }{ }^{5} \text { and } \\
\text { [FIPS 202]. }\end{array}$ \\
\hline Key-derivation function & $\begin{array}{l}\text { As used in this Recommendation, either a one-step key-derivation } \\
\text { method or a key-derivation function based on a pseudorandom } \\
\text { function as specified in [SP } 800-108] \text {. }\end{array}$ \\
\hline Key-derivation method & $\begin{array}{l}\text { As used in this Recommendation, a process that derives secret } \\
\text { keying material from a shared secret. This Recommendation } \\
\text { specifies both one-step and two-step key-derivation methods. }\end{array}$ \\
\hline Key-derivation procedure & $\begin{array}{l}\text { As used in this Recommendation, a two-step key-derivation method } \\
\text { consisting of randomness extraction followed by key expansion. }\end{array}$ \\
\hline Key-derivation key & $\begin{array}{l}\text { As used in this Recommendation, a key that is used during the key- } \\
\text { expansion step of a key-derivation procedure to derive the secret } \\
\text { output keying material. This key-derivation key is obtained from a } \\
\text { shared secret during the randomness-extraction step. }\end{array}$ \\
\hline Key establishment & $\begin{array}{l}\text { A procedure that results in secret keying material that is shared } \\
\text { among different parties. }\end{array}$ \\
\hline Key expansion & $\begin{array}{l}\text { The second step in the key-derivation procedure specified in this } \\
\text { Recommendation in which a key-derivation key is used to derive } \\
\text { secret keying material having the desired length. }\end{array}$ \\
\hline Keying material & $\begin{array}{l}\text { Data that is represented as a binary string such that any non- } \\
\text { overlapping segments of the string with the required lengths can be } \\
\text { used as secret keys, secret initialization vectors, and other secret } \\
\text { parameters. }\end{array}$ \\
\hline $\begin{array}{l}\text { Message Authentication } \\
\text { Code (MAC) algorithm }\end{array}$ & $\begin{array}{l}\text { A family of cryptographic functions that is parameterized by a } \\
\text { symmetric key. Each of the functions can act on input data (called } \\
\text { a "message") of variable length to produce an output value of a } \\
\text { specified length. The output value is called the MAC of the input } \\
\text { message. MAC }(k, x, \ldots) \text { is used to denote the MAC of message } x \\
\text { computed using the key } k \text { (and any additional algorithm-specific } \\
\text { parameters). An approved MAC algorithm is expected to satisfy } \\
\text { the following property (for each supported security strength): }\end{array}$ \\
\hline
\end{tabular}

${ }^{5}$ FIPS 180, Secure Hash Standard (SHS)

${ }^{6}$ FIPS 202, SHA-3 Standard: Permutation-Based Hash and Extendable-Output Functions 


\begin{tabular}{|c|c|}
\hline & $\begin{array}{l}\text { Without knowledge of the key } k \text {, it must be computationally } \\
\text { infeasible to predict the (as-yet-unseen) value of } \operatorname{MAC}(k, x, \ldots) \\
\text { with a probability of success that is a significant improvement } \\
\text { over simply guessing either the MAC value or } k \text {, even if one has } \\
\text { already seen the results of using that same key to compute } \\
\text { MAC }\left(k, x_{j}, \ldots\right) \text { for (a bounded number of) other messages } x_{j} \neq x \text {. } \\
\text { A MAC algorithm can be employed to provide authentication of the } \\
\text { origin of data and/or to provide data-integrity protection. In this } \\
\text { Recommendation, approved MAC algorithms are used to } \\
\text { determine families of pseudorandom functions (indexed by the } \\
\text { choice of key) that may be employed during key derivation. }\end{array}$ \\
\hline Nonce & $\begin{array}{l}\text { A varying value that has, at most, a negligible chance of } \\
\text { repeating; for example, a random value that is generated anew for } \\
\text { each use, a timestamp, a sequence number, or some combination } \\
\text { of these. }\end{array}$ \\
\hline $\begin{array}{l}\text { Pseudorandom function } \\
\text { family (PRF) }\end{array}$ & $\begin{array}{l}\text { An indexed family of (efficiently computable) functions, each } \\
\text { defined for the same particular pair of input and output spaces. (For } \\
\text { the purposes of this Recommendation, one may assume that both } \\
\text { the index set and the output space are finite.) The indexed functions } \\
\text { are pseudorandom in the following sense: } \\
\text { If a function from the family is selected by choosing an index } \\
\text { value uniformly at random, and one's knowledge of the } \\
\text { selected function is limited to the output values corresponding } \\
\text { to a feasible number of (adaptively) chosen input values, then } \\
\text { the selected function is computationally indistinguishable from } \\
\text { a function whose outputs were fixed uniformly at random. }\end{array}$ \\
\hline Randomness extraction & $\begin{array}{l}\text { The first step in the two-step key-derivation procedure specified in } \\
\text { this Recommendation; during this step, a key-derivation key is } \\
\text { produced from a shared secret. }\end{array}$ \\
\hline Salt & $\begin{array}{l}\text { As used in this Recommendation, a byte string (which may be } \\
\text { secret or non-secret) that is used as a MAC key by either: 1) a } \\
\text { MAC-based auxiliary function } \mathrm{H} \text { employed in one-step key } \\
\text { derivation or 2) a MAC employed in the randomness-extraction } \\
\text { step during two-step key derivation. }\end{array}$ \\
\hline Security strength & $\begin{array}{l}\text { A number characterizing the amount of work that is expected to } \\
\text { suffice to "defeat" an implemented cryptographic mechanism (e.g., } \\
\text { by compromising its functionality and/or circumventing the } \\
\text { protection that its use was intended to facilitate). In this } \\
\text { Recommendation, security strength is measured in bits. If the } \\
\text { security strength of a particular implementation of a cryptographic }\end{array}$ \\
\hline
\end{tabular}




\begin{tabular}{|c|c|}
\hline & $\begin{array}{l}\text { mechanism is } s \text { bits, it is expected that the equivalent of (roughly) } \\
2^{s} \text { basic operations of some sort will be sufficient to defeat it in } \\
\text { some way. }\end{array}$ \\
\hline Shared secret & $\begin{array}{l}\text { The secret byte string that is computed/generated during the } \\
\text { execution of an approved key-establishment scheme and used as } \\
\text { input to a key-derivation method as part of that transaction. }\end{array}$ \\
\hline Shall & $\begin{array}{l}\text { A requirement that needs to be fulfilled to claim conformance to } \\
\text { this Recommendation. Note that shall may be coupled with not to } \\
\text { become shall not. }\end{array}$ \\
\hline $\begin{array}{l}\text { Support } \\
\text { (a security strength) }\end{array}$ & $\begin{array}{l}\text { A security strength of } s \text { bits is said to be supported by a particular } \\
\text { choice of algorithm, primitive, auxiliary function, or parameters for } \\
\text { use in the implementation of a cryptographic mechanism if that } \\
\text { choice will not prevent the resulting implementation from attaining } \\
\text { a security strength of at least } s \text { bits. } \\
\text { In this Recommendation, it is assumed that implementation choices } \\
\text { are intended to support a security strength of } 112 \text { bits or more (see } \\
\left.[\text { SP } 800-57]^{7} \text { and [SP } 800-131 \mathrm{~A}\right]^{8} \text { ). }\end{array}$ \\
\hline Symmetric key & $\begin{array}{l}\text { A single cryptographic key that is used with a symmetric-key } \\
\text { algorithm; also called a secret key. A symmetric-key algorithm is a } \\
\text { cryptographic algorithm that uses the same secret key for an } \\
\text { operation and its complement (e.g., encryption and decryption). }\end{array}$ \\
\hline Targeted security strength & $\begin{array}{l}\text { The security strength that is intended to be supported by one or } \\
\text { more implementation-related choices (such as algorithms, } \\
\text { primitives, auxiliary functions, parameter sizes, and/or actual } \\
\text { parameters) for the purpose of implementing a cryptographic } \\
\text { mechanism. }\end{array}$ \\
\hline
\end{tabular}

${ }^{7}$ SP 800-57 Rev. 4, Recommendation for Key Management Part 1: General

${ }^{8}$ SP 800-131A, Transitions: Recommendation for Transitioning the Use of Cryptographic Algorithms and Key Lengths 


\subsection{Symbols and Abbreviations}

\begin{tabular}{|c|c|}
\hline $0 \mathrm{x}$ & $\begin{array}{l}\text { A marker used to indicate that the following symbols are to be } \\
\text { interpreted as a bit string written in hexadecimal notation (using } \\
\text { the symbols } 0,1, \ldots, 9 \text { and } \mathrm{A}, \mathrm{B}, \ldots, \mathrm{F} \text { to denote } 4 \text {-bit binary } \\
\text { representations of the integers zero through nine and } 10 \text { through } \\
15 \text {, respectively). A byte can be represented by a hexadecimal } \\
\text { string of length two; the leftmost hexadecimal symbol corresponds } \\
\text { to the most significant four bits of the byte, and the rightmost } \\
\text { hexadecimal symbol corresponds to the least significant four bits } \\
\text { of the byte. For example, 0x9D represents the bit string } 10011101 \\
\text { (assuming that the bits are positioned in order of decreasing } \\
\text { significance). }\end{array}$ \\
\hline AES & $\begin{array}{l}\text { Advanced Encryption Standard (the block cipher specified in } \\
\text { [FIPS 197] }]^{9} \text { ). }\end{array}$ \\
\hline $\begin{array}{l}\text { AES- } N \\
(N=128,192, \text { or } 256)\end{array}$ & $\begin{array}{l}\text { The variant of the AES block cipher that requires an } N \text {-bit } \\
\text { encryption/decryption key; the three variants specified in [FIPS } \\
\text { 197] are AES-128, AES-192, and AES-256. }\end{array}$ \\
\hline AES-CMAC & $\begin{array}{l}\text { The Cipher-based Message Authentication Code (CMAC) mode } \\
\text { of operation for the AES block cipher, as specified in [ㄹ 800- } \\
\underline{38 \mathrm{~B}}]^{10} \text {. }\end{array}$ \\
\hline $\begin{array}{l}\mathrm{AES}-N-\mathrm{CMAC}(k, x) \\
(N=128,192, \text { or } 256)\end{array}$ & $\begin{array}{l}\text { An implementation of AES-CMAC based on the AES- } N \text { variant of } \\
\text { the AES block cipher (for } N=128,192 \text {, or 256); its output is a } \\
\text { 128-bit MAC computed over the "message" } x \text { using the key } k \text {. }\end{array}$ \\
\hline counter & $\begin{array}{l}\text { An unsigned integer, represented as a big-endian four-byte string, } \\
\text { that is employed by the one-step key-derivation method specified } \\
\text { in Section } 4.1 \text {. }\end{array}$ \\
\hline Context & $\begin{array}{l}\text { A bit string of context-specific data; a subcomponent of the } \\
\text { FixedInfo that is included as part of the input to the two-step key- } \\
\text { derivation method specified in Section 5.1. }\end{array}$ \\
\hline default_salt & $\begin{array}{l}\text { A default value assigned to salt (if necessary) to implement an } \\
\text { auxiliary function } \mathrm{H} \text { selected according to Option } 2 \text { or } 3 \text { in the one- } \\
\text { step key-derivation method specified in Section } 4.1 \text {. }\end{array}$ \\
\hline
\end{tabular}

${ }^{9}$ FIPS 197, Advanced Encryption Standard

${ }^{10}$ SP 800-38B, Recommendation for Block Cipher Modes of Operation: the CMAC Mode for Authentication 


\begin{tabular}{|c|c|}
\hline DerivedKeyingMaterial & $\begin{array}{l}\text { Keying material that is derived from a shared secret } Z \text { (and other } \\
\text { data) through the use of a key-derivation method. }\end{array}$ \\
\hline ECC & Elliptic curve cryptography. \\
\hline FFC & Finite field cryptography. \\
\hline FixedInfo & $\begin{array}{l}\text { A bit string of context-specific data whose value does not change } \\
\text { during the execution of a key-derivation method specified in this } \\
\text { Recommendation. }\end{array}$ \\
\hline $\mathrm{H}$ & $\begin{array}{l}\text { The auxiliary function used to produce blocks of keying material } \\
\text { during the execution of the one-step key-derivation method specified } \\
\text { in Section 4.1. }\end{array}$ \\
\hline hash & $\begin{array}{l}\text { A hash function. Approved choices for hash are specified in [FIPS } \\
\text { 180] and [FIPS 202]. }\end{array}$ \\
\hline HMAC & $\begin{array}{l}\text { Keyed-hash Message Authentication Code, as specified in [FIPS } \\
\text { 198]. }{ }^{11}\end{array}$ \\
\hline HMAC-hash $(k, x)$ & $\begin{array}{l}\text { An implementation of HMAC using the hash function, hash; its } \\
\text { output is a MAC computed over "message" } x \text { using the key } k \text {. }\end{array}$ \\
\hline H_outputBits & $\begin{array}{l}\text { A positive integer that indicates the length (in bits) of the output of } \\
\text { either: 1) the auxiliary function } \mathrm{H} \text { used in the one-step key- } \\
\text { derivation method specified in Section } 4.1 \text { or } 2 \text { ) an auxiliary } \\
\text { HMAC algorithm used in the two-step key-derivation method } \\
\text { specified in Section 5.1. }\end{array}$ \\
\hline IFC & Integer factorization cryptography. \\
\hline$I V$ & $\begin{array}{l}\text { Initialization vector; as used in this Recommendation, it is a bit } \\
\text { string used as an initial value during the execution of an approved } \\
\text { PRF-based KDF operating in Feedback Mode, as specified in [SP } \\
\underline{800-108]} \text {. }\end{array}$ \\
\hline $\mathrm{KDF}$ & Key-derivation function. \\
\hline$K_{D K}$ & $\begin{array}{l}\text { The key-derivation key resulting from the randomness-extraction } \\
\text { step, which is then used in the key-expansion step during the } \\
\text { execution of the key-derivation procedure specified in Section 5.1. }\end{array}$ \\
\hline
\end{tabular}

${ }^{11}$ FIPS 198, The Keyed-Hash Message Authentication Code (HMAC) 


\begin{tabular}{|c|c|}
\hline$K D M$ & Key-derivation method. \\
\hline KMAC & $\begin{array}{l}\text { Keccak Message Authentication Code, as specified in } \\
\text { [SP 800-185]. }{ }^{12}\end{array}$ \\
\hline $\operatorname{KMAC\# }(k, x, l, S)$ & $\begin{array}{l}\text { A variant of KMAC (either KMAC } 128 \text { or KMAC } 256 \text {, as specified } \\
\text { in [SP 800-185]); its output is an } l \text {-bit MAC computed over the } \\
\text { "message" } x \text { using the key } k \text { and "customization string" } S \text {. }\end{array}$ \\
\hline$L$ & $\begin{array}{l}\text { A positive integer specifying the desired length (in bits) of the } \\
\text { derived keying material. }\end{array}$ \\
\hline$[L]_{2}$ & An agreed-upon encoding of the integer $L$ as a bit string. \\
\hline MAC & Message Authentication Code. \\
\hline $\operatorname{MAC}(k, x, \ldots)$ & $\begin{array}{l}\text { An instance of a MAC algorithm computed over the "message" } x \\
\text { using the key } k \text { (and any additional algorithm-specific parameters). }\end{array}$ \\
\hline max_H_inputBits & $\begin{array}{l}\text { The maximum length (in bits) for strings used as input to the } \\
\text { auxiliary function } \mathrm{H} \text { employed by the one-step key-derivation } \\
\text { method specified in Section } 4.1 \text {. }\end{array}$ \\
\hline OtherInput & $\begin{array}{l}\text { A collective term for any and all additional data (other than the } \\
\text { shared secret itself) that is used as input to a key-derivation method } \\
\text { specified in this Recommendation. }\end{array}$ \\
\hline PRF & Pseudorandom function (family). \\
\hline$s$ & Security strength (in bits). \\
\hline SHA & $\begin{array}{l}\text { Secure Hash Algorithm, as specified in [FIPS 180] (i.e., SHA-1, } \\
\text { SHA-224, SHA-512/224, SHA-256, SHA-512/256, SHA-384, or } \\
\text { SHA-512) or [FIPS 202] (i.e., SHA3-224, SHA3-256, SHA3-384, } \\
\text { or SHA3-512). }\end{array}$ \\
\hline$Z$ & $\begin{array}{l}\text { The shared secret (determined as described in Section } 2 \text { of this } \\
\text { Recommendation). }\end{array}$ \\
\hline
\end{tabular}

${ }^{12}$ SP 800-185, SHA-3 Derived Functions: cSHAKE, KMAC, TupleHash, and ParallelHash 


\section{$4 \quad$ One-Step Key Derivation}

This section specifies a family of approved key-derivation functions (KDFs) that are executed in a single step; a two-step procedure is specified in Section 5. The input to each specified KDF includes the shared secret $Z$, an indication of the desired bit length of the keying material to be output, and, perhaps, other information (as determined by the particular implementation of the keyestablishment scheme and/or key-derivation function).

Implementations of these one-step KDFs depend upon the choice of an auxiliary function $\mathrm{H}$, which can be either: 1) an approved hash function, denoted as hash, as defined in [FIPS 180] or [FIPS 202]; 2) HMAC with an approved hash function, hash, denoted as HMAC-hash and defined in [FIPS 198]; or 3) a KMAC variant, as defined in [SP 800-185]. Tables 1, 2, and 3 in Section 4.2 describe the possibilities for $\mathrm{H}$ and also include any restrictions on the associated implementationdependent parameters. $\mathrm{H}$ shall be chosen in accordance with the selection requirements specified in Section 7.

When an approved MAC algorithm (HMAC or KMAC) is used to define the auxiliary function $\mathrm{H}$, it is permitted to use a known salt value as the MAC key. In such cases, it is assumed that the MAC algorithm will satisfy the following property (for each of its supported security strengths):

Given knowledge of the key $k$, and (perhaps) partial knowledge of a message $x$ that includes an unknown substring $y$, it must be computationally infeasible to predict the (as-yet-unseen) value of $\operatorname{MAC}(k, x, \ldots)$ with a probability of success that is a significant improvement over simply guessing either the MAC value or the value of $y$, even if one has already seen the values of $\operatorname{MAC}\left(k_{j}, x_{j}, \ldots\right)$ for a feasible number of other $\left(k_{j}, x_{j}\right)$ pairs where each key $k_{j}$ is known and each (partially known) message $x_{j}$ includes the same unknown substring $y$, provided that none of the $\left(k_{j}, x_{j}\right)$ pairs is identical to $(k, x)$.

This property is consistent with the use of the MAC algorithm as the specification of a family of pseudorandom functions defined on the appropriate message space and indexed by the choice of MAC key. Under Option 2 and Option 3 of the KDF specification below, the auxiliary function $\mathrm{H}$ is a particular selection from such a family. The (partially known) messages will have the form counter $\|Z\|$ FixedInfo, containing the shared secret $Z$ as an unknown substring.

\subsection{Specification of Key-Derivation Functions}

A family of one-step key-derivation functions is specified as follows:

Function call: $\operatorname{KDM}(Z$, OtherInput $)$.

\section{Options for the Auxiliary Function H:}

Option 1: $\mathrm{H}(x)=$ hash $(x)$, where hash is an approved hash function meeting the selection requirements specified in Section 7 , and the input, $x$, is a bit string.

Option 2: $\mathrm{H}(x)=\mathrm{HMAC}-h a s h($ salt, $x)$, where HMAC-hash is an implementation of the HMAC algorithm (as defined in [FIPS 198]) employing an approved hash function, hash, that meets the selection requirements specified in Section 7. An implementationdependent byte string, salt, whose (non-null) value may be optionally provided in 
OtherInput, serves as the HMAC key, and $x$ (the input to $\mathrm{H}$ ) is a bit string that serves as the HMAC "message" as specified in [FIPS 198].

Option 3: $\mathrm{H}(x)=\mathrm{KMAC \#}($ salt, $x, H$ _outputBits, $S)$, where KMAC\# is a particular implementation of either KMAC128 or KMAC256 (as defined in [SP 800-185]) that meets the selection requirements specified in Section 7. An implementationdependent byte string, salt, whose (non-null) value may be optionally provided in OtherInput, serves as the KMAC\# key, and $x$ (the input to $\mathrm{H}$ ) is a bit string that serves as the KMAC\# "message" as specified in [SP 800-185]. The parameter H_outputBits determines the bit length chosen for the output of the KMAC variant employed. The "customization string" $S$ shall be the byte string 01001011 || 01000100 || 01000110 , which represents the sequence of characters "K", "D," and "F" in 8-bit ASCII. (This three-byte string is denoted by "KDF" in this document.)

\section{Implementation-Dependent Parameters:}

1. H_outputBits - A positive integer that indicates the length (in bits) of the output of the auxiliary function $\mathrm{H}$ that is used to derive blocks of secret keying material. If Option 1 or Option 2 is chosen, then H_outputBits corresponds to the bit-length of the output block of the particular hash function used in the implementation of $\mathrm{H}$; therefore, H_outputBits is in the set $\{160,224,256,384,512\}$ with the precise value determined by the choice for the hash function, hash (see Section 4.2 for details). If Option 3 is chosen, then H_outputBits shall either be set equal to the length (in bits) of the secret keying material to be derived (see input $L$ below) or selected from the set $\{160,224,256,384,512\}$.

2. max_H_inputBits - A positive integer that indicates the maximum permitted length (in bits) of the bit string $x$ that is used as input to the auxiliary function H. If Option 1 or Option 2 is chosen for the implementation of $\mathrm{H}$, then the upper bound on max_H_inputBits shall not

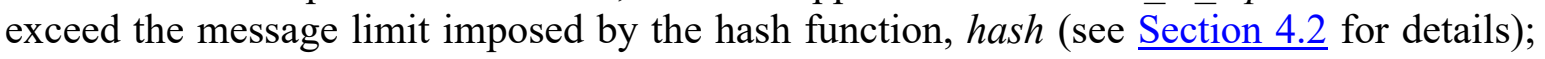
max_H_inputBits values smaller than a specification-imposed upper bound may be dictated by the particular use case. If the hash function, hash, is specified in [FIPS 202], or if Option 3 is chosen for the implementation of $\mathrm{H}$, then there is no specification-imposed upper bound on max_H_inputBits; the value assigned to max_H_inputBits may be determined by the needs of the relying applications/parties.

3. default_salt-A non-null (secret or non-secret) byte string that is needed only if either Option 2 (HMAC-hash) or Option 3 (KMAC\#) is chosen for the implementation of the auxiliary function $\mathrm{H}$. This byte string is used as the value of salt if a (non-null) value is not included in OtherInput (see below).

If $\mathrm{H}(x)=$ HMAC-hash (salt, $x)$, then - in the absence of an agreed-upon alternative - the default_salt shall be an all-zero byte string whose bit length equals that specified as the bit length of an input block for the hash function, hash. (Input-block lengths for the approved hash functions that can be employed to implement HMAC-hash are listed in Table 1 of Section 4.2.)

If $\mathrm{H}(x)=\mathrm{KMAC} 128$ (salt, $x, H_{-}$outputBits, "KDF"), then - in the absence of an agreed-upon alternative - the default_salt shall be an all-zero string of 164 bytes (i.e., an all-zero string of 1312 bits). 
If $\mathrm{H}(x)=\mathrm{KMAC} 256$ (salt, $x, H$ _outputBits, "KDF"), then - in the absence of an agreed-upon alternative - the default_salt shall be an all-zero string of 132 bytes (i.e., an all-zero string of 1056 bits).

\section{Input:}

1. $Z$ - a byte string that represents the shared secret.

2. OtherInput, which includes:

a. $\{$ salt $\}$ - A non-null (secret or non-secret) byte string that can be (optionally) provided if either Option 2 (HMAC-hash) or Option 3 (KMAC\#) is chosen for the implementation of the auxiliary function $\mathrm{H}$ since those options require a salt value that is used as a MAC key.

The salt included in OtherInput could be, for example, a value computed from nonces exchanged as part of a key-establishment protocol that employs one or more of the keyagreement schemes specified in [SP 800-56A] or [SP 800-56B], a value already shared by the protocol participants, or a value that is pre-determined by the protocol. The possibilities for the length of salt are determined as follows:

(1) The HMAC-hash algorithm, as defined in [FIPS 198], can accommodate MAC keys of any bit length permitted for input to the hash function, hash. Therefore, when Option 2 is chosen, the length of the byte string salt can be as large as allowed for any string used as input to hash. However, if the bit length of salt is greater than the bit length specified for a single input block for the hash function, hash, then the value of salt is replaced by hash(salt) as part of the HMAC computation. See Table 2 for details.

(2) The KMAC128 and KMAC256 algorithms specified in [SP 800-185] can accommodate MAC keys of any length up to $\left(2^{2040}-1\right)$ bits. Therefore, when Option 3 is chosen, salt can be a byte string of any agreed-upon length that does not exceed ( $2^{2037}-1$ bytes) (i.e., $2^{2040}-8$ bits). The input salt value will be (re)formatted (using a byte-padding function) during the execution of the KMAC algorithm to obtain a string whose length is a multiple of either 168 bytes (for KMAC128) or 136 bytes (for KMAC256). See Table 3 for details.

If a salt value required by $\mathrm{H}$ is omitted from OtherInput (or if a required salt value included in OtherInput is the null string), then the value of default_salt shall be used as the value of salt when $\mathrm{H}$ is executed.

b. $L-$ A positive integer that indicates the length (in bits) of the secret keying material to be derived; $L$ shall not exceed $H \_$outputBits $\times\left(2^{32}-1\right)$.

( $L=$ keydatalen in the notation of previous versions of [SP 800-56A], while $L=K B i t s$ in the notation of previous versions of [SP 800-56B] ; current versions of those documents have been updated to be consistent with SP 800-56C.)

c. FixedInfo - A bit string of context-specific data that is appropriate for the relying keyestablishment scheme. As its name suggests, the value of FixedInfo does not change during the execution of the process described below.

FixedInfo may, for example, include appropriately formatted representations of the 
values of salt and/or $L$. The inclusion of additional copies of the values of salt and $L$ in FixedInfo would ensure that each block of derived keying material is affected by all of the information conveyed in OtherInput. See [SP 800-56A] and [SP 800-56B] for more detailed recommendations concerning the format and content of FixedInfo (also known as OtherInfo in earlier versions of those documents).

\section{Process:}

1. If $L>0$, then set reps $=\left\lceil L / H \_\right.$outputBits $\rceil$; otherwise, output an error indicator and exit this process without performing the remaining actions (i.e., omit steps 2 through 8 ).

2. If reps $>\left(2^{32}-1\right)$, then output an error indicator and exit this process without performing the remaining actions (i.e., omit steps 3 through 8 ).

3. Initialize a big-endian 4-byte unsigned integer counter as $0 \mathrm{x} 00000000$, corresponding to a 32-bit binary representation of the number zero.

4. If counter $\|Z\|$ FixedInfo is more than max_H_inputBits bits long, then output an error indicator and exit this process without performing any of the remaining actions (i.e., omit steps 5 through 8).

5. Initialize Result(0) as an empty bit string (i.e., the null string).

6. For $i=1$ to reps, do the following:

6.1 Increment counter by 1.

6.2 Compute $K(i)=\mathrm{H}($ counter $\|Z\|$ FixedInfo $)$.

6.3 Set $\operatorname{Result}(i)=\operatorname{Result}(i-1) \| K(i)$.

7. Set DerivedKeyingMaterial equal to the leftmost $L$ bits of Result(reps).

8. Output DerivedKeyingMaterial.

Output:

The bit string DerivedKeyingMaterial of length $L$ bits (or an error indicator).

\section{Notes:}

In step 6.2 above, if $\mathrm{H}(x)=$ hash $(x)$ or $\mathrm{H}(x)=\mathrm{HMAC}-h a s h($ salt, $x)$, the entire output block of the hash function, hash, shall be used when computing the output of H. Some approved choices for hash (e.g., SHA-512/224, SHA-512/256, and SHA-384, as specified in [FIPS 180]) include an internal truncation operation. In such a case, the "entire output" of hash is the output block as defined in its specification. (For example, in the case of hash = SHA-384, the entire output is defined as a 384-bit block resulting from the internal truncation of a certain 512-bit value).

If $\mathrm{H}(x)=\mathrm{KMAC} \#\left(\right.$ salt $, x, H \_$outputBits, $\left.S\right)$, then choosing $H \_$outputBits $=L$ will likely be the most efficient way to produce the desired $L$ bits of keying material.

The derived keying material DerivedKeyingMaterial shall be computed in its entirety before outputting any portion of it. 


\subsection{The Auxiliary Function $\mathrm{H}(\boldsymbol{x})$ and Related Parameters}

Tables $\underline{1}, \underline{2}$, and $\underline{3}$ enumerate the possibilities for the auxiliary function $\mathrm{H}$ and provide additional information concerning the values of related parameters, such as H_outputBits and max_H_inputBits. The tables also indicate the range of security strengths that can be supported by each choice for $\mathrm{H}$ (see Section 4.1) when used in a key derivation function for a key-establishment scheme specified in $\underline{\text { SP 800-56A }}$ or SP 800-56B.

Table 1: $\mathbf{H}(x)=\operatorname{hash}(x) \quad(O p t i o n ~ 1)$

\begin{tabular}{|c|c|c|c|c|}
\hline $\begin{array}{c}\text { Hash } \\
\text { Function } \\
\text { (hash) }\end{array}$ & $\begin{array}{c}\text { Byte / Bit } \\
\text { Length of } \\
\text { Input Blocks }\end{array}$ & $\begin{array}{c}\text { H_outputBits } \\
\text { (in bits) }\end{array}$ & $\begin{array}{c}\text { max_H_inputBits } \\
\text { (in bits) }\end{array}$ & $\begin{array}{l}\text { Security } \\
\text { Strength } s \\
\text { supported } \\
\text { (in bits) }\end{array}$ \\
\hline SHA-1 & $64 / 512$ & 160 & \multirow{3}{*}{$\leq 2^{64}-1$} & $112 \leq s \leq 160$ \\
\hline SHA-224 & 64 / 512 & 224 & & $112 \leq s \leq 224$ \\
\hline SHA-256 & 64 / 512 & 256 & & $112 \leq s \leq 256$ \\
\hline SHA-512/224 & $128 / 1024$ & 224 & \multirow{4}{*}{$\leq 2^{128}-1$} & $112 \leq s \leq 224$ \\
\hline SHA-512/256 & $128 / 1024$ & 256 & & $112 \leq s \leq 256$ \\
\hline SHA-384 & $128 / 1024$ & 384 & & $112 \leq s \leq 384$ \\
\hline SHA-512 & $128 / 1024$ & 512 & & $112 \leq s \leq 512$ \\
\hline SHA3-224 & $144 / 1152$ & 224 & \multirow{4}{*}{$\begin{array}{l}\text { Arbitrarily long } \\
\text { inputs can be } \\
\text { accommodated. }\end{array}$} & $112 \leq s \leq 224$ \\
\hline SHA3-256 & $136 / 1088$ & 256 & & $112 \leq s \leq 256$ \\
\hline SHA3-384 & $104 / 832$ & 384 & & $112 \leq s \leq 384$ \\
\hline SHA3-512 & $72 / 576$ & 512 & & $112 \leq s \leq 512$ \\
\hline
\end{tabular}

Table 2: H(x) = HMAC-hash(salt, $x)$ (Option 2)

\begin{tabular}{|c|c|c|c|c|}
\hline $\begin{array}{c}\text { Hash } \\
\text { Function } \\
\text { (hash) }\end{array}$ & $\begin{array}{c}\text { Effective } \\
\text { Byte / Bit } \\
\text { Length* } \\
\text { of salt }\end{array}$ & $\begin{array}{c}\text { H_outputBits } \\
\text { (in bits) }\end{array}$ & $\begin{array}{c}\text { max_H_inputBits } \\
\text { (in bits) }\end{array}$ & $\begin{array}{l}\text { Security } \\
\text { Strength } s \\
\text { supported } \\
\text { (in bits) }\end{array}$ \\
\hline SHA-1 & $64 / 512$ & 160 & \multirow{3}{*}{$\leq 2^{64}-513$} & $112 \leq s \leq 160$ \\
\hline SHA-224 & $64 / 512$ & 224 & & $112 \leq s \leq 224$ \\
\hline SHA-256 & $64 / 512$ & 256 & & $112 \leq s \leq 256$ \\
\hline SHA-512/224 & $128 / 1024$ & 224 & \multirow{4}{*}{$\leq 2^{128}-1025$} & $112 \leq s \leq 224$ \\
\hline SHA-512/256 & $128 / 1024$ & 256 & & $112 \leq s \leq 256$ \\
\hline SHA-384 & $128 / 1024$ & 384 & & $112 \leq s \leq 384$ \\
\hline SHA-512 & $128 / 1024$ & 512 & & $112 \leq s \leq 512$ \\
\hline SHA3-224 & $144 / 1152$ & 224 & Arbitrarily long & $112 \leq s \leq 224$ \\
\hline
\end{tabular}




\begin{tabular}{|l|c|c|c|c|}
\hline SHA3-256 & $136 / 1088$ & 256 & \multirow{2}{*}{$\begin{array}{c}\text { inputs can be } \\
\text { accommodated. }\end{array}$} & $112 \leq s \leq 256$ \\
\cline { 1 - 2 } SHA3-384 & $104 / 832$ & 384 & & $112 \leq s \leq 384$ \\
\cline { 1 - 2 } \cline { 1 - 2 } SHA3-512 & $72 / 576$ & 512 & & $112 \leq s \leq 512$ \\
\hline
\end{tabular}

* This Recommendation places no restriction on the length of a chosen salt other than the requirement that its byte length be greater than zero but no greater than the length of a single input block to the hash function, hash, used to implement HMAC-hash. That freedom of choice is somewhat illusory, however, since the HMAC algorithm will convert an input salt value (as needed) into a string of the indicated hash-dependent length. A shorter salt (used by $\mathrm{H}$ as an HMAC key) will be padded by appending an all-zero bit string to obtain a string of the indicated length (the length of a single input block for the hash function, hash); a longer salt will be hashed to produce a shorter string (of bit length an all-zero bit string) to obtain a string of the indicated length (see [FIPS 198] for additional information).

Table 3: H(x) = KMAC\#(salt, $x$, H_outputBits, “KDF") (Option 3)

\begin{tabular}{|c|c|c|c|c|c|}
\hline $\begin{array}{l}\text { KMAC } \\
\text { Variant }\end{array}$ & $\begin{array}{l}\text { Length } \\
\text { of the } \\
\text { encoded } \\
\text { salt value }\end{array}$ & $\begin{array}{l}\text { Suggested } \\
\text { Maximum } \\
\text { Byte Length } \\
\text { of salt }\end{array}$ & $\begin{array}{c}\text { H_outputBits } \\
\text { (in bits) }\end{array}$ & $\begin{array}{c}\text { max_H_inputBits } \\
\text { (in bits) }\end{array}$ & $\begin{array}{l}\text { Security } \\
\text { Strength } s \\
\text { supported } \\
\text { (in bits) }\end{array}$ \\
\hline KMAC128 & $\begin{array}{l}\text { Multiple } \\
\text { of } 168 \\
\text { bytes }\end{array}$ & $\begin{array}{c}168-4= \\
164 * *\end{array}$ & \multirow{2}{*}{$\begin{array}{c}\text { Choice of } 160 \text {, } \\
224,256,384 \text {, } \\
512 \text {, or } L .\end{array}$} & \multirow{2}{*}{$\begin{array}{l}\text { Arbitrarily long } \\
\text { inputs can be } \\
\text { accommodated. }\end{array}$} & $112 \leq s \leq 128$ \\
\hline KMAC256 & $\begin{array}{l}\text { Multiple } \\
\text { of } 136 \\
\text { bytes }\end{array}$ & $\begin{array}{r}136-4= \\
132 * * *\end{array}$ & & & $112 \leq s \leq 256$ \\
\hline
\end{tabular}

** KMAC\# prepends a length encoding for the first input data field. For KMAC128, using 164 bytes (or less) of salt leaves room for 4 bytes of prepended length encoding and limits the length of the encoded salt to no more than the length of a single block of input to KMAC128.

*** KMAC\# prepends a length encoding for the first input data field. For KMAC256, using 132 bytes (or less) of salt leaves room for 4 bytes of prepended length encoding and limits the length of the encoded salt to no more than the length of a single block of input to KMAC256. 


\section{Two-Step Key Derivation}

This section specifies an approved (two-step) extraction-then-expansion key-derivation procedure. Like the one-step key-derivation functions described in Section 4 , the input to this twostep procedure includes the shared secret $Z ; L$, a positive integer indicating the desired length (in bits) of the output keying material; and other information (as determined by the particular implementation of the key-establishment scheme and/or key-derivation method). In contrast to the one-step methods, a salt value is required to be included as part of the input.

The extraction-then-expansion key-derivation procedure is pictured in Figure 1.

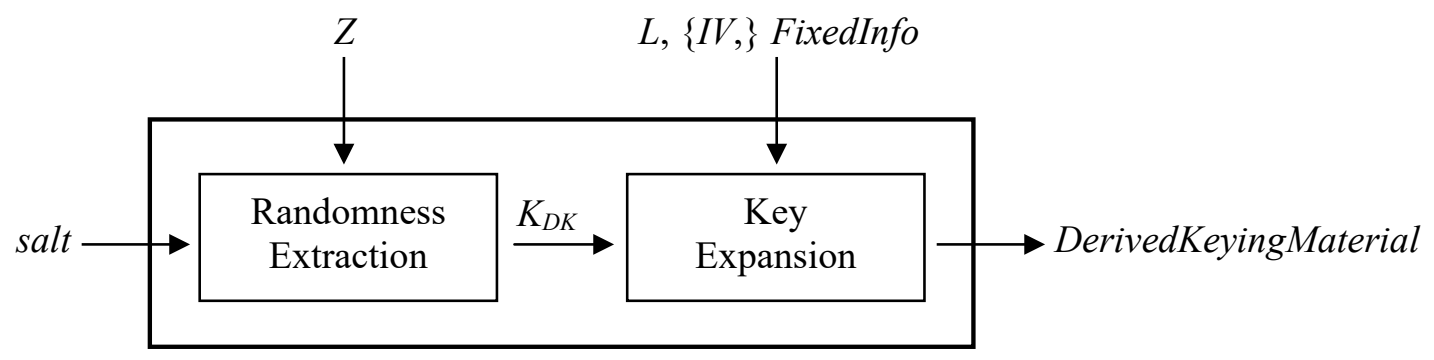

Figure 1: The Extraction-then-Expansion Key-Derivation Procedure

The first (randomness-extraction) step uses either HMAC (as defined in [FIPS 198]) or AESCMAC (as defined in [SP 800-38B]). In either case, there are two inputs: salt, which serves as a MAC key and the shared secret, $Z$, which serves as the "message." The resulting MAC output is used as a key-derivation key, $K_{D K}$. The use of the $K_{D K}$ shall be restricted to the execution of the key-expansion step of the (two step) key-derivation procedure in which the $K_{D K}$ is extracted from $Z$. In particular, its value shall not be an output of the extraction-then-expansion key-derivation procedure.

The second (key-expansion) step uses the key-derivation key, $K_{D K}$, along with the integer $L$ and other appropriate data as the input to a PRF-based key-derivation function specified in [SP 800108]. The output returned by that key-derivation function is either secret keying material (in the form of DerivedKeyingMaterial, a bit string of length $L$ ) or an error indicator.

\subsection{Specification of Key-Derivation Procedure}

The extraction-then-expansion key-derivation procedure is specified as follows:

\section{Function call: $\operatorname{KDM}(Z$, OtherInput $)$.}

\section{Options for the Auxiliary MAC Algorithm:}

The MAC algorithm employed for randomness extraction shall be either an implementation of HMAC as defined in [FIPS 198], based on an approved hash function, hash (i.e., HMAChash), or an implementation of AES-CMAC as defined in [SP 800-38B] (i.e., AES- $N$-CMAC for $N=128,192$, or 256). In either case, the (untruncated) output of the MAC algorithm is used as the key-derivation key for subsequent key expansion. Tables 4 and 5 in Section 5.2 
describe the possibilities for the auxiliary MAC algorithm, which shall be chosen in accordance with the selection requirements specified in Section 7.

\section{Implementation-Dependent Auxiliary PRF-based KDF:}

One of the general-purpose, PRF-based key-derivation functions defined in [SP 800-108] shall be used for key expansion. These key-derivation functions employ an approved MAC algorithm as the PRF. In this Recommendation, the PRF used by the KDF in key expansion is determined by the MAC algorithm that is used for randomness extraction. Specifically:

a. If HMAC-hash is used in the randomness-extraction step, then the same HMAC-hash (i.e., using the same hash function, hash) shall be used as the PRF in the key-expansion step; and

b. If AES-128-CMAC, AES-192-CMAC, or AES-256-CMAC is used in the randomnessextraction step, then only AES-128-CMAC (i.e., the CMAC mode of AES-128) shall be used as the PRF in the key-expansion step.

The rationale for these rules is discussed in Section 8.3.

\section{Input:}

1. $Z$ - A byte string that represents the shared secret. It is used as the "message" during the execution of the MAC algorithm employed in the randomness-extraction step.

2. OtherInput, which includes:

a. salt - A non-null (secret or non-secret) byte string used as the MAC key during the execution of the randomness-extraction step (i.e., step 1 in the process shown below). This salt could be, for example, a value computed from nonces exchanged as part of a keyestablishment protocol that employs one or more of the key-agreement schemes specified in [SP 800-56A] or [SP 800-56B], a value already shared by the protocol participants, or a value that is pre-determined by the protocol. The possibilities for the length of salt are determined by the auxiliary MAC algorithm that is used for randomness extraction:

(1) The HMAC-hash algorithm as defined in [FIPS 198] can accommodate keys of any length up to the maximum bit length permitted for input to the hash function, hash. Therefore, the length of the byte string salt can be as large as allowed for any string used as input to hash. However, if the bit length of salt is greater than the bit length specified for a single input block for hash, then the value of salt is replaced by hash(salt) as part of the HMAC computation. (Input-block lengths for the approved hash functions that can be employed to implement HMAC-hash are included in column 4 of Table 1 in Section 4.2; also see Table 4 of Section 5.2.) In the absence of an agreedupon alternative, the input salt value shall be an all-zero byte string whose length is equal to that of a single input block for the hash function, hash.

(2) AES- $N$-CMAC requires keys that are $N$ bits long (for $N=128,192$, or 256 ), depending upon the AES variant that is used in the implementation. The bit length of salt shall be 
the bit length required of a key for that AES variant (128 bits for AES-128, 192 bits for AES-192, or 256 bits for AES-256). In the absence of an agreed-upon alternative, the input salt value shall be an all-zero string of the required bit length.

b. $L-$ A positive integer that indicates the length (in bits) of the secret keying material to be derived using the auxiliary PRF-based KDF during the execution of the key-expansion step (i.e., step 2 in the process shown below). The maximum value allowed for $L$ is determined by the mode (i.e., Counter Mode, Feedback Mode, or Double-Pipeline Iteration Mode) and implementation details of the chosen KDF (as specified in [SP 800-108]). An error event will occur during the execution of the KDF if $L$ is too large. ${ }^{13}$

(Note that $L=$ keydatalen in the notation of previous versions of [SP 800-56A], while $L=$ KBits in the notation of previous versions of [SP 800-56B]; current versions of those documents have been updated to be consistent with SP 800-56C.)

c. $\{I V\}-$ A bit string included (if required) for use as an initial value during an execution of the auxiliary PRF-based KDF; an $I V$ shall be included in OtherInput if and only if the chosen PRF-based KDF is operating in Feedback Mode. It can either be secret or nonsecret. It may be an empty string. If the PRF-based KDF is operating in either Counter Mode or Double-Pipeline Iteration Mode, an $I V$ shall not be included in OtherInput. (See [SP 800-108] for details.)

d. FixedInfo, including:

(1) Label - A bit string that identifies the purpose for the derived keying material. For example, it can be the ASCII encoding of a character string describing the relying application(s) and/or the intended use(s) of the keying material. The value and encoding method used for the Label are defined in a larger context, for example, in the protocol that uses this key-derivation procedure. As an alternative to including this string as a separate component of FixedInfo, Label could be incorporated in Context (see below).

(2) Context - A bit string of context-specific data appropriate for the relying keyestablishment scheme/protocol and the chosen PRF-based KDF.

For recommendations concerning the format and context-specific content of Context, see the specifications of FixedInfo and/or OtherInfo in [SP 800-56A] and/or [SP 80056B], respectively.

(3) $[L]_{2}$ - An agreed-upon encoding of $L$ as a bit string that is appropriate for use by the chosen PRF-based KDF (see [SP 800-108] for details). As an alternative to including this string as a separate component of FixedInfo, $[L]_{2}$ could be incorporated in Context (see above).

\footnotetext{
${ }^{13}$ The restrictions on the size of $L$ that are given in [SP 800-108] are stated in terms of $n=\lceil L / h\rceil$, where $h$ denotes the bit length of an output block of the PRF used to implement the auxiliary KDF. In the case of Counter Mode, the restriction is $n \leq 2^{r}-1$, where $r \leq 32$ is the (implementation-dependent) bit length allocated for the KDF's counter variable. For the other KDF modes, the restriction is simply $n \leq 2^{32}-1$.
} 


\section{Process:}

\section{[Randomness Extraction]}

1. Call MAC $($ salt $, Z, \ldots)$ to obtain $K_{D K}$ or an error indicator. If an error occurs, output an error indicator, and exit from this process without performing step 2.

\section{[Key Expansion]}

2. Call $\mathrm{KDF}\left(K_{D K}, L,\{I V\right.$,$\left.\} FixedInfo \right)$ to obtain DerivedKeyingMaterial or an error indicator (see [SP 800-108] for details). If an error occurs, output an error indicator; otherwise output DerivedKeyingMaterial.

\section{Output:}

The bit string DerivedKeyingMaterial of length $L$ bits (or an error indicator).

\section{Notes:}

When HMAC-hash is used as the auxiliary MAC algorithm, the length of $K_{D K}$ is the length of an untruncated output block from the hash function, hash. When AES-CMAC is used, then (regardless of the AES variant employed) $K_{D K}$ is a 128-bit binary string. $K_{D K}$ is used (locally) as a key-derivation key by the auxiliary KDF during the key-expansion step and shall be destroyed (along with all other sensitive, locally stored data) after its use. Its value shall not be an output of the key-derivation procedure.

[RFC 5869] specifies a version of the above extraction-then-expansion key-derivation procedure using HMAC for both the extraction and expansion steps. For an extensive discussion concerning the rationale for the extract-and-expand mechanisms specified in this Recommendation, see [LNCS 6223].

\subsection{The Auxiliary MAC Algorithm and Related Parameters}

Tables $\underline{4}$ and $\underline{5}$ enumerate the possibilities for the auxiliary MAC algorithm used for randomness extraction and provide additional information concerning the lengths of the MAC key (i.e., the salt value) and the extracted key-derivation key (i.e., $K_{D K}$ ). The tables also indicate the range of security strengths that can be supported by each choice for MAC (see Section 5.1) when used for

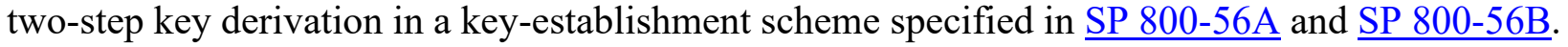

Table 4: MAC( salt, Z,...) = HMAC-hash( salt, Z ) (For Randomness Extraction)

\begin{tabular}{|l|c|c|c|}
\hline $\begin{array}{c}\text { Hash } \\
\text { Function } \\
\text { (hash) }\end{array}$ & $\begin{array}{c}\text { Effective } \\
\text { Byte / Bit } \\
\text { Length* } \\
\text { of salt }\end{array}$ & $\begin{array}{c}\text { Bit Length of } \\
\text { Extracted } \boldsymbol{K}_{\boldsymbol{D K}}\end{array}$ & $\begin{array}{c}\text { Security } \\
\text { Strength } \boldsymbol{s} \\
\text { supported } \\
\text { (in bits) }\end{array}$ \\
\hline SHA-1 & $64 / 512$ & 160 & $112 \leq s \leq 160$ \\
\hline SHA-224 & $64 / 512$ & 224 & $112 \leq s \leq 224$ \\
\hline SHA-256 & $64 / 512$ & 256 & $112 \leq s \leq 256$ \\
\hline
\end{tabular}




\begin{tabular}{|l|c|c|c|}
\hline SHA-512/224 & $128 / 1024$ & 224 & $112 \leq s \leq 224$ \\
\hline SHA-512/256 & $128 / 1024$ & 256 & $112 \leq s \leq 256$ \\
\hline SHA-384 & $128 / 1024$ & 384 & $112 \leq s \leq 384$ \\
\hline SHA-512 & $128 / 1024$ & 512 & $112 \leq s \leq 512$ \\
\hline SHA3-224 & $144 / 1152$ & 224 & $112 \leq s \leq 224$ \\
\hline SHA3-256 & $136 / 1088$ & 256 & $112 \leq s \leq 256$ \\
\hline SHA3-384 & $104 / 832$ & 384 & $112 \leq s \leq 384$ \\
\hline SHA3-512 & $72 / 576$ & 512 & $112 \leq s \leq 512$ \\
\hline
\end{tabular}

* This Recommendation places no restriction on the length of a chosen salt other than the requirement that its byte length be greater than zero but no greater than the length of a single input block to the hash function, hash, used to implement HMAC-hash. That freedom of choice is somewhat illusory, however, since the HMAC algorithm will convert an input salt value (as needed) into a string of the indicated hash-dependent length. A shorter salt (which is used as an HMAC key) will be padded (by appending an all-zero bit string) to obtain a string of the indicated length (the length of a single input block for the hash function, hash); a longer salt will be hashed to produce a shorter string, which will then be padded (by appending an all-zero bit string) to obtain a string of the indicated length. (See [FIPS 198] for additional information.)

Note: The hash function, hash, used by the HMAC algorithm employed during randomness extraction shall be used again in the subsequent key-expansion step to implement the HMAC algorithm that is employed as a PRF by the auxiliary PRF-based KDF.

Table 5: MAC( salt, $Z, \ldots)=$ AES- $N$-CMAC( salt, $Z$ ) (For Randomness Extraction)

\begin{tabular}{|l|c|c|c|}
\hline $\begin{array}{c}\text { AES Variant } \\
\text { used by } \\
\text { AES-CMAC }\end{array}$ & $\begin{array}{c}\text { Bit Length } \\
\text { of salt for } \\
\text { AES-CMAC }\end{array}$ & $\begin{array}{c}\text { Bit Length of } \\
\text { Extracted } \boldsymbol{K}_{\boldsymbol{D K}}\end{array}$ & $\begin{array}{c}\text { Security } \\
\text { Strength } \boldsymbol{s} \\
\text { supported } \\
\text { (in bits) }\end{array}$ \\
\cline { 1 - 2 } AES-128 & 128 & \multirow{2}{*}{128} & \multirow{2}{*}{$112 \leq s \leq 128$} \\
\cline { 1 - 2 } AES-192 & 192 & & \\
\hline AES-256 & 256 &
\end{tabular}

Note: Regardless of which AES variant is used by the AES-CMAC algorithm during randomnessextraction, the 128-bit AES block size determines the bit length of the resulting $K_{D K}$ and the maximum security strength that can be supported by the use of the derived keying material (i.e., no more than 128 bits). To accommodate the use of this 128 -bit $K_{D K}$ as a key-derivation key, the CMAC mode of AES-128 shall be the PRF employed by the auxiliary PRF-based KDF in the subsequent key-expansion step.

\subsection{Randomness Extraction followed by Multiple Key Expansions}

The two-step key-derivation procedure specified in Section 5.1 can be generalized to incorporate 
a single instance of randomness extraction followed by $m$ instances of key expansion for some (implementation-dependent) integer $m \geq 2$.

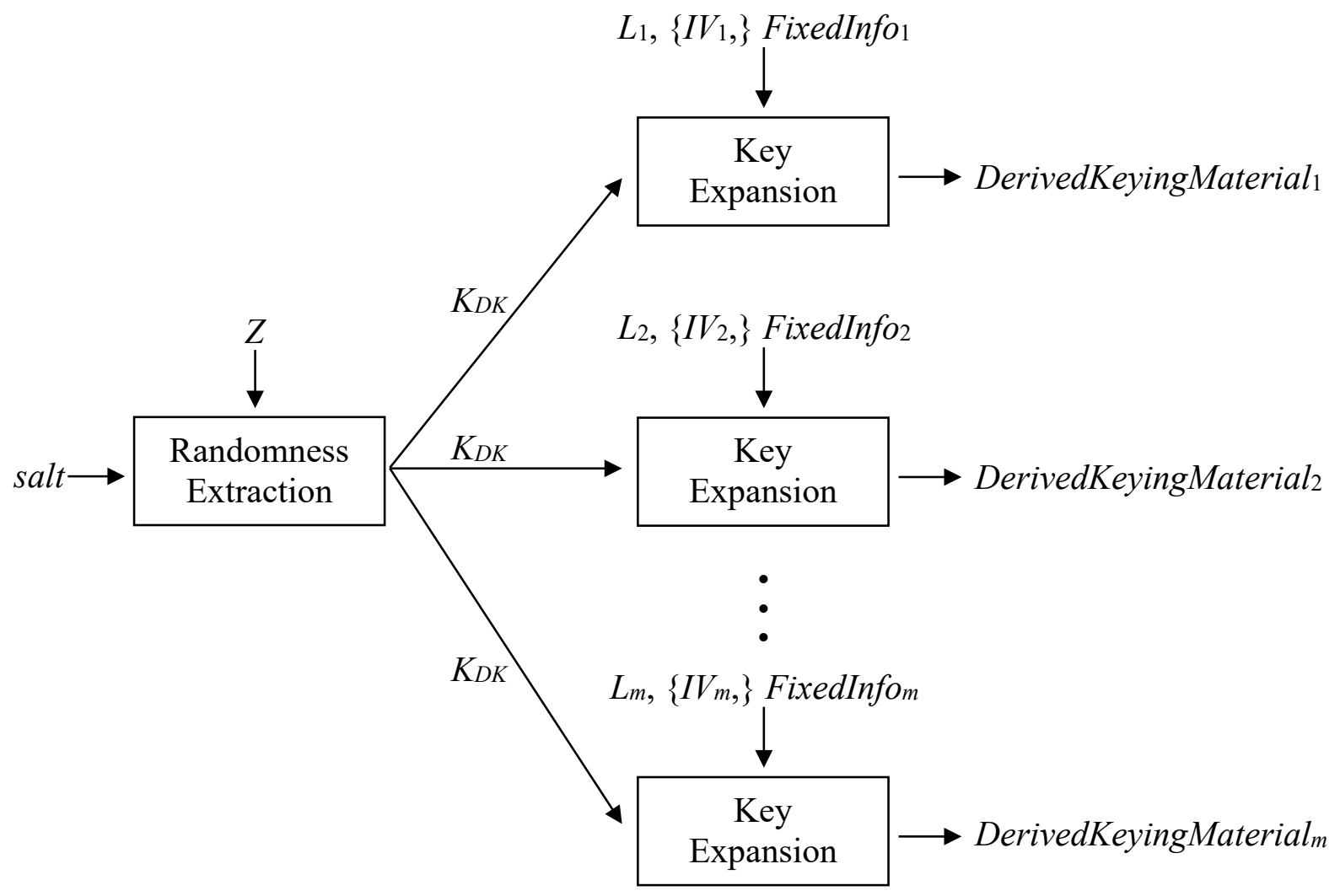

Figure 2: Randomness Extraction followed by Multiple Key Expansions

For conformance with this Recommendation, the following restrictions apply:

- The auxiliary MAC algorithm employed for randomness extraction shall be either an implementation of HMAC as defined in [FIPS 198], based on an approved hash function, hash (i.e., HMAC-hash), or an implementation of AES-CMAC as defined in [SP 800-38B] (i.e., AES- $N$-CMAC for $N=128,192$, or 256). In either case, the (untruncated) output of the MAC algorithm shall be used as the key-derivation key for subsequent key expansion. Tables 4 and 5 in Section 5.2 describe the possibilities for the auxiliary MAC algorithm, which shall be chosen in accordance with the selection requirements specified in Section 7.

- One of the general-purpose, PRF-based key-derivation functions defined in [SP 800-108] shall be used for key expansion. The same KDF shall be used to implement all $m$ expansion operations. In particular, the same key-derivation mode (counter mode, feedback mode, or double-pipeline iteration mode) and the same PRF shall be employed by the KDF in each of the $m$ keyexpansion operations.

- The PRF used by the KDF in key expansion is determined by the MAC algorithm that is used for randomness extraction. Specifically:

a. If HMAC-hash is used for randomness extraction, then the same HMAC-hash (i.e., using 
the same hash function, hash) shall be the PRF used by the KDF in key expansion.

and

b. If either AES-128-CMAC, AES-192-CMAC, or AES-256-CMAC is used for randomness extraction, then the PRF used by the KDF in key-expansion shall be AES-128-CMAC (i.e., the CMAC mode of AES-128).

- The OtherInput provided during the key-derivation request shall provide the salt for the randomness-extraction step (see Section 5.1 for additional details), and the requisite inputs (other than the key-derivation key) for $m$ calls to the PRF-based KDF used for key expansion. In particular, for $i=1, . ., m$, OtherInput shall include (subject to the stated conditions):

a. $L_{i}-$ A positive integer that indicates the length (in bits) of the secret keying material to be derived during the $i$-th call to the PRF-based KDF. (See the description of $L$ in Section 5.1 for additional details.)

b. $\quad\left\{I V_{i}\right\}-\mathrm{A}$ bit string included (if required) for use as an initial value for the $i$-th call to the PRF-based KDF; the $I V_{i}$ values shall be included in OtherInput if and only if the chosen PRF-based KDF is operating in Feedback Mode. (See the description of $I V$ in Section 5.1 for additional details.)

c. FixedInfo - The FixedInfo data to be employed during the $i$-th call to the PRF-based KDF. (See the description of FixedInfo in Section 5.1 for details.)

- The values of FixedInfo1, FixedInfo2, .., and FixedInfom shall be (pairwise) distinct. (See Section 7.5, item 2 in [SP 800-108].)

- The derived keying material, DerivedKeyingMaterial 1, DerivedKeyingMaterial $_{2}, \ldots$, and

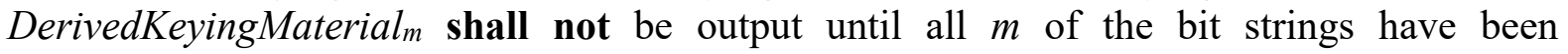
successfully computed. If an error occurs during randomness extraction or key expansion, then this key-derivation method shall not output any derived keying material.

To incorporate $m$ key-expansion operations into an extract-then-expand key-derivation procedure, the process and output specified in Section 5.1 are modified as follows:

\section{Process:}

\section{[Randomness Extraction]}

1. Call MAC $($ salt $, Z, \ldots)$ to obtain $K_{D K}$ or an error indicator; if an error occurs, output an error indicator and exit from this process without performing steps 2 and 3.

\section{[Key Expansion]}

2. For $i=1$ to $m$, do the following:

2.1 Call KDF( $K_{D K}, L_{i},\left\{I V_{i},\right\}$ FixedInfo $\left._{i}\right)$ to obtain DerivedKeyingMaterial $i$ or an error indicator (see [SP 800-108] for details). If an error occurs, output an error indicator and exit this process without performing any of the remaining actions (in particular, omit step 3).

3. For $i=1$ to $m$, do the following: 


\subsection{Output DerivedKeyingMaterial . $_{\text {. }}$}

\section{Output:}

The bit strings DerivedKeyingMaterial, DerivedKeyingMaterial $2, \ldots$, and DerivedKeyingMaterial $l_{m}$ of lengths $L_{1}$ bits, $L_{2}$ bits, ... , and $L_{m}$ bits, respectively (or an error indicator).

\section{Notes:}

As specified in Section 5.1: When HMAC-hash is used as the auxiliary MAC algorithm, the length of $K_{D K}$ is the length of an untruncated output block from the hash function, hash. When AES-CMAC is used, then (regardless of the AES variant employed) $K_{D K}$ is a 128-bit binary string. The extracted $K_{D K}$ is used (locally) as a key-derivation key by the auxiliary KDF during key expansion (step 2 above) and shall be destroyed (along with all other sensitive locally stored data) after its use. Its value shall not be an output of the key-derivation procedure. 


\section{$6 \quad$ Application-Specific Key-Derivation Methods}

Additional approved, application-specific key-derivation methods are enumerated in [SP 800-135]. Unless an explicit exception is made in [SP 800-135], any hash function or MAC algorithm employed by the key-derivation methods enumerated in [SP 800-135] shall be approved and shall also meet the selection requirements specified in this Recommendation (i.e., SP 800-56C). 


\section{Selecting Hash Functions and MAC Algorithms}

The key-derivation methods specified in this Recommendation, as well as those enumerated in [ $\underline{\mathrm{SP}}$ 800-135], use hash functions and/or message authentication code (MAC) algorithms as auxiliary functions. In particular:

- The one-step key-derivation functions that are specified in Section 4.1 of this Recommendation employ an appropriate choice of hash function (hash), an HMAC algorithm based on an appropriate choice of hash function (HMAC-hash), or one of two KMAC variants (KMAC128 or KMAC256) to implement the auxiliary function $\mathrm{H}$.

- The extraction-then-expansion key-derivation procedure specified in Section 5.1 employs either an HMAC algorithm based on an appropriate choice of hash function (HMAC-hash) for both randomness extraction and key expansion or an appropriate variant of the AESCMAC algorithm (i.e., AES- $N$-CMAC for $N=128,192$, or 256 ) for randomness extraction together with AES-128-CMAC for key expansion.

Unless explicitly stated to the contrary (e.g., in [SP 800-135]), the following requirements apply to the hash functions and MAC algorithms employed for key derivation:

- Whenever a hash function is employed (including as the primitive used by HMAC), an approved hash function shall be used. [FIPS 180] and [FIPS 202] specify approved hash functions.

- Whenever an HMAC algorithm is employed, the HMAC implementation shall conform to the specifications found in [FIPS 198].

- Whenever a KMAC variant (KMAC128 or KMAC256) is employed, the KMAC implementation shall conform to the specifications found in [SP 800-185].

- Whenever an AES-CMAC algorithm is employed, the implementation of AES shall conform to [FIPS 197], and the AES-CMAC implementation shall conform to [SP 80038B].

As specified in [SP 800-56A] and [SP 800-56B], an approved key-establishment scheme can be implemented with parameters of various types and sizes that will impact the estimated maximum security strength that can be supported by the resulting scheme. When a key-establishment scheme employs a choice of parameters that are associated with a targeted security strength of $s$ bits, the selection of a hash function, HMAC, KMAC, or AES-CMAC employed during the implementation of its key-derivation method shall conform to the following restrictions:

- An approved hash function shall be employed (whether alone or as the primitive used by HMAC) in the implementation of a one-step or two-step key-derivation method only if its output block length (in bits) is greater than or equal to $s$.

- For the purposes of implementing one-step key derivation only: KMAC128 shall be employed only in instances where $s$ is 128 bits or less. KMAC256 shall be employed only in instances where $s$ is 256 bits or less. (However, see the note below.) 
- For the purposes of implementing two-step key derivation only: AES-CMAC shall be employed only in instances where $s$ is 128 bits or less. (See the note following Table 5.)

Tables 1 through 5 (in Sections 4.1 and 5.1) can be consulted to determine which hash functions and/or MAC algorithms are approved for use when a key-derivation method specified in this Recommendation is used by an approved key-establishment scheme to support a targeted security strength of $s$ bits.

Note: At the time of publication of this Recommendation, a key-establishment scheme implemented in accordance with either [SP $800-56 \mathrm{~A}]$ or $[\mathrm{SP} 800-56 \mathrm{~B}]$ can have a targeted security strength of 256 bits at most. 


\section{$8 \quad$ Further Discussion}

In this section, the following issues are discussed:

\subsection{Using a Truncated Hash Function}

SHA-224, SHA-512/224, SHA-512/256, and SHA-384 are among the approved hash functions specified in [FIPS 180]. SHA-224 is a truncated version of SHA-256, while SHA-512/224, SHA512/256, and SHA-384 are truncated versions of SHA-512. (Each of these truncated versions uses a specific initial chaining value, which is different from the initial chaining value used by the untruncated version.) In applications that require a relatively long bit string of derived keying material, implementing the key-derivation methods specified in this Recommendation with a truncated version of a hash function may be less efficient than using the corresponding untruncated version (i.e., SHA-256 or SHA-512).

\subsection{The Choice of a Salt Value}

In this Recommendation, the MAC algorithms employed either in a one-step key-derivation method or in the randomness-extraction step of a two-step key-derivation method use a salt value as a MAC key (see Sections $\underline{4}$ and $\underline{5}$ ). This Recommendation does not require the use of a randomly selected salt value. In particular, if there are no means to select a salt value and share it with all of the participants during a key-establishment transaction, then this Recommendation specifies that a predetermined default (e.g., all-zero) byte string be used as the salt value. The benefits of using "random" salt values when possible are discussed (briefly) in Section 3.1 ("To salt or not to salt") of [RFC 5869] and in greater detail in [LNCS 6223].

\subsection{MAC Algorithms used for Extraction and Expansion}

Provided that the targeted security strength can be supported (see Tables 4 and 5 in Section 5.2), this Recommendation permits either HMAC-hash (i.e., HMAC implemented with an appropriately chosen approved hash function, hash) or AES-CMAC (i.e., the CMAC mode of AES-128, AES192, or AES-256) to be selected as the MAC algorithm used in the randomness-extraction step of the key-derivation procedure specified in Section 5.1.

The PRF-based KDF used in the key-expansion step of the procedure also requires an appropriate MAC (to serve as the PRF). While it may be technically feasible (in some cases) to employ completely different MAC algorithms in the two steps of the specified key-derivation procedure, this Recommendation does not permit such flexibility. Instead, the following restrictions have been placed on MAC selection (see Sections $\underline{5}$ and $\underline{7}$ ):

- When HMAC-hash is chosen for use in the randomness-extraction step, the same MAC algorithm (i.e., HMAC-hash with the same approved hash function, hash) shall be employed to implement the PRF-based KDF used in the key-expansion step.

- When AES-128-CMAC, AES-192-CMAC, or AES-256-CMAC is chosen for use in the randomness-extraction step, the MAC algorithm employed by the PRF-based KDF used in the key-expansion step shall be AES-128-CMAC, the CMAC mode of AES-128. (AES- 
128 is the only AES variant that can employ the 128 -bit $K_{D K}$ produced by AES- $N$-CMAC during the randomness-extraction step.)

- The MAC algorithm selected for the implementation of a two-step key-derivation method shall be capable of supporting the targeted security strength as determined by consulting Tables 4 and 5 in Section 5.2. (This limits the use of AES-CMAC to cases where the targeted security strength is no more than 128 bits.)

The imposed restrictions are intended to reduce the overall complexity of the resulting implementations, promote interoperability, and simplify the negotiation of the parameters and auxiliary functions affecting the security strength supported by the key-derivation procedure.

Note: At this time, KMAC has not been specified for use in the implementation of a two-step keyderivation procedure. This restriction may be reconsidered once a KMAC-based KDF has been approved for use as a PRF-based KDF in a revision of [SP 800-108].

\subsection{Destruction of Sensitive Locally Stored Data}

Good security practice dictates that implementations of key-derivation methods include steps that destroy potentially sensitive locally stored data that is created (and/or copied for use) during the execution of a particular process; there is no need to retain such data after the process has been completed. Examples of potentially sensitive locally stored data include local copies of shared secrets that are employed during the execution of a particular process, intermediate results produced during computations, and locally stored duplicates of values that are ultimately output by the process. The destruction of such locally stored data ideally occurs prior to or during any exit from the process. This is intended to limit opportunities for unauthorized access to sensitive information that might compromise a key-establishment transaction.

It is not possible to anticipate the forms of all possible implementations of the key-derivation methods specified in this Recommendation, making it equally impossible to enumerate all potentially sensitive data that might be locally stored by a process employed in a particular implementation. Nevertheless, the destruction of any potentially sensitive locally stored data is an obligation of all implementations. 


\section{References}

[FIPS 180] National Institute of Standards and Technology (2015) Secure Hash Standard (SHS). (U.S. Department of Commerce, Washington, DC), Federal Information Processing Standards Publication (FIPS) 180-4.

https://doi.org/10.6028/NIST.FIPS.180-4

[FIPS 197] National Institute of Standards and Technology (2001) Advanced Encryption Standard (AES). (U.S. Department of Commerce, Washington, DC), Federal Information Processing Standards Publication (FIPS) 197. https://doi.org/10.6028/NIST.FIPS.197

[FIPS 198] National Institute of Standards and Technology (2008) The Keyed-Hash Message Authentication Code (HMAC). (U.S. Department of Commerce, Washington, DC), Federal Information Processing Standards Publication (FIPS) 198-1.

https://doi.org/10.6028/NIST.FIPS.198-1

[FIPS 202] National Institute of Standards and Technology (2015) SHA-3 Standard: Permutation-Based Hash and Extendable-Output Functions. (U.S. Department of Commerce, Washington, DC), Federal Information Processing Standards Publication (FIPS) 202. https://doi.org/10.6028/NIST.FIPS.202

[LNCS 6223] Krawczyk H (2010) Cryptographic Extraction and Key Derivation: The HKDF Scheme. Advances in Cryptology - Crypto'2010, ed. Rabin T (Springer, Berlin, Germany), Lecture Notes in Computer Science vol. 6223, pp 631-648. https://doi.org/10.1007/978-3-642-14623-7_34

[RFC 5869] Krawczyk H, Eronen P (2010) HMAC-based Extract-and-Expand Key Derivation Function (HKDF). (Internet Engineering Task Force (IETF)), IETF Request for Comments (RFC) 5869.

https://doi.org/10.17487/RFC5869

[SP 800-38B] Dworkin MJ (2005) Recommendation for Block Cipher Modes of Operation: the CMAC Mode for Authentication. (National Institute of Standards and Technology, Gaithersburg, MD), NIST Special Publication (SP) 800-38B, Includes updates as of October 6, 2016.

https://doi.org/10.6028/NIST.SP.800-38B

[SP 800-56A] Barker EB, Chen L, Roginsky A, Davis R (2018) Recommendation for PairWise Key-Establishment Schemes Using Discrete Logarithm Cryptography. ((National Institute of Standards and Technology, Gaithersburg, MD), NIST Special Publication (SP) 800-56A, Rev. 3. https://doi.org/10.6028/NIST.SP.800-56Ar3 
[SP 800-56B] Barker EB, Chen L, Roginsky A, Vassilev A, Davis R, Simon S (2019) Recommendation for Pair-Wise Key-Establishment Using Integer Factorization Cryptography. (National Institute of Standards and Technology, Gaithersburg, MD), NIST Special Publication (SP) 800-56B, Rev. 2. https://doi.org/10.6028/NIST.SP.800-56Br2

[SP 800-57] Barker EB (2020) Recommendation for Key Management, Part 1: General. (National Institute of Standards and Technology, Gaithersburg, MD), Draft NIST Special Publication (SP) 800-57 Part 1, Rev. 5. https://doi.org/10.6028/NIST.SP.800-57pt1r5

[SP 800-108] Chen L (2009) Recommendation for Key Derivation Using Pseudorandom Functions (Revised). (National Institute of Standards and Technology, Gaithersburg, MD), NIST Special Publication (SP) 800-108, Revised. https://doi.org/10.6028/NIST.SP.800-108

[SP 800-131A] Barker EB, Roginsky A (2019) Transitioning the Use of Cryptographic Algorithms and Key Lengths. (National Institute of Standards and Technology, Gaithersburg, MD), NIST Special Publication (SP) 800-131A, Rev. 2. https://doi.org/10.6028/NIST.SP.800-131Ar2

[SP 800-135] Dang QH (2011) Recommendation for Existing Application-Specific Key Derivation Functions. (National Institute of Standards and Technology, Gaithersburg, MD), NIST Special Publication (SP) 800-135, Rev. 1. https://doi.org/10.6028/NIST.SP.800-135r1

[SP 800-185] Kelsey JM, Chang S-jH, Perlner RA (2016) SHA-3 Derived Functions: cSHAKE, KMAC, TupleHash, and ParallelHash. (National Institute of Standards and Technology, Gaithersburg, MD), NIST Special Publication (SP) 800-185. https://doi.org/10.6028/NIST.SP.800-185 


\section{Appendix A: Revisions (Informative)}

\section{A.1 The Original Version of SP 800-56C}

The original SP 800-56C (published in November 2011) focused entirely on the specification of a two-step extraction-then-expansion key-derivation procedure to be used in conjunction with a keyestablishment scheme from either [SP 800-56A] or [SP 800-56B]. It provided an alternative to the one-step key-derivation functions that were already included in those companion publications.

\section{A.2 Revision 1}

The 2018 revision of SP 800-56C reorganized the original content (it still included the specification of an extraction-then-expansion key-derivation procedure) to also include the specification of a family of one-step key-derivation functions, expanding on material that was previously found only in SP 800-56A and SP 800-56B. This change was made in support of the removal of detailed descriptions of key-derivation methods from SP 800-56A and a future revision of SP 800-56B. The consolidation of specifications in SP 800-56C, Revision 1 promoted consistency between the key-derivation options available for use with an approved keyestablishment scheme chosen from either of those companion NIST publications. (A number of application-specific key-derivation methods specified in [SP 800-135] continued to be supported.)

Specifically named key-establishment "parameter sets" (FA - FC for finite-field cryptography (FFC); EA - EE for elliptic-curve cryptography (ECC); and IA - IB for integer-factorization cryptography (IFC)) were no longer used as guides for choosing the auxiliary functions employed by a key-derivation method. Instead, SP 800-56C, Revision 1 indicated the security strengths that could be supported by the various possibilities for the auxiliary functions. Implementers were expected to let the targeted security strength of the key-establishment scheme guide their choices. Of course, each of the named parameter sets was associated with a targeted security strength, so this was more a change of perspective rather than of substance. The change was, however, consistent with the revision of [SP 800-56A], which de-emphasized (in the FFC case) or eliminated (in the ECC case) the use of named parameter (size) sets.

There was one substantial change to the specification of key-derivation methods that is worth noting: a KMAC-based option for implementing the auxiliary function $\mathrm{H}$ was added to the specification of one-step key-derivation functions (see Section 4.1). At that time, however, KMAC had not been specified for use as an auxiliary MAC algorithm in the two-step extraction-thenexpansion key-derivation procedure (see Section 8.3).

Given the extent to which SP 800-56C had been revised, it is impractical to list all of the changes that were made to the original text. It is recommended that SP $800-56 \mathrm{C}$, Revision 1 be read in its entirety in order to gain familiarity with the details of the current specifications for both the onestep and two-step key-derivation methods used in approved key-establishment schemes.

\section{A.3 Revision 2}

The 2020 revision of SP $800-56 C$ involves just a few changes to the 2018 version of the document. 
In Section 2, the applicability of the various key-derivation methods specified in this Recommendation is expanded to permit the use of "hybrid" shared secrets of the form $Z^{\prime}=Z \| T$, which is a concatenation consisting of a "standard" shared secret $Z$ that was generated during the execution of a key-establishment scheme as currently specified in [SP 800-56A] or [SP 800-56B], followed by an auxiliary shared secret $T$ that has been generated using some other method.

This is not a substantive change in the case of one-step key-derivation methods, which derive blocks of keying material from input of the form counter $\|Z\|$ FixedInfo. Implementations of approved key-establishment schemes have considerable latitude concerning the content and format of the context-specific data included in FixedInfo. Replacing $Z$ with $Z^{\prime}=Z \| T$ is equivalent to replacing FixedInfo with FixedInfo $o^{\prime}=T \|$ FixedInfo, which was already permitted. As in previous versions of this document, $T$ could instead be used as a salt value by the auxiliary function $\mathrm{H}$ (see Option 2 and Option 3) and/or included in some (other) subfield of FixedInfo. (See Section $\underline{4.1}$ and Section 4.2 for details.)

In the case of the two-step key-derivation methods, the extraction of a key-derivation key from a shared secret of the form $Z \| T$ is a bona fide extension of the previously specified technique but is still consistent with the principles of randomness extraction and key expansion as presented in [LNCS 6223]. Prior to this revision, $T$ could only have been included either as a salt value (in an HMAC-based extraction step) or as part of the FixedInfo used in the key-expansion step. (See $\underline{\text { Section } 5.1}$ and Section 5.2 for details.)

The other change made in the 2020 revision affects the key-expansion step of the approved twostep key-derivation methods. The newly added Section 5.3 specifies the conditions under which multiple instances of key expansion can be performed using a single key-derivation key obtained via randomness extraction.

A specific change: In Section 3.1, the definition for hash function was modified to include all three properties often required of a cryptographic hash function and to state that the pre-image resistance of a hash function is a contributing factor when determining the security strength provided by a key-derivation method. 